\title{
Short communication: Calf cleanliness does not predict diarrhea upon arrival at a veal calf facility
}

\author{
A. N. Graham, D. L. Renaud, T. F. Duffield, and D. F. Kelton ${ }^{1}$ \\ Department of Population Medicine, University of Guelph, Ontario, N1G 2W1, Canada
}

\section{ABSTRACT}

The objective of this study was to validate the use of cleanliness scores to identify the presence of diarrhea in calves. On arrival at a milk-fed veal facility, 452 calves were scored for hide cleanliness and fecal consistency by 1 of 2 observers. Fecal consistency was scored on a scale of 0 to 3 , where fecal score of $0=$ normal consistency, 1 $=$ semiformed or pasty, $2=$ loose feces, and $3=$ watery feces; calves with a fecal score of 2 or 3 were classified as positive for diarrhea. Hide cleanliness was also scored on a scale of 0 to 3 , where $0=$ clean thighs and body with little to no manure on lower legs; $1=$ tail head region and back end of calf are soiled with manure; $2=$ tail head region, back end of calf, and thighs or legs are soiled with manure; and $3=$ tail head region, back end of calf, thighs, and legs are soiled with manure. Of the calves scored, 188 calves (42\%) were identified as having diarrhea based on hide cleanliness; however, only 78 calves $(17 \%)$ were identified with diarrhea based on fecal consistency. The level of agreement between the 2 scoring methods were calculated, and a weighted kappa of 0.22 indicated only fair agreement between the 2 scoring methods. However, the sensitivity and specificity, calculated using fecal consistency $\geq 2$ as the classification variable, were 67 and $63 \%$, respectively, when a cut point of $\geq 1$ for cleanliness score was used. A total of 222 calves scored at arrival were scored once per day for an additional $2 \mathrm{~d}$ following arrival. Calves were more likely to have more days with abnormal hide cleanliness than abnormal fecal consistency; 91 calves (41\%) had an abnormal cleanliness score for at least $2 \mathrm{~d}$, whereas only 21 calves (9\%) had an abnormal fecal score for at least $2 \mathrm{~d}$. We found poor correlation between total number of days with an abnormal cleanliness score and total number of days with an abnormal fecal score, indicating that consecutive observations of hide cleanliness would not improve the validity of using

Received November 8, 2017.

Accepted January 2, 2018.

${ }^{1}$ Corresponding author: dkelton@uoguelph.ca hide cleanliness. Thus, hide cleanliness is not a good indicator for identifying diarrhea in calves, and scoring fecal samples for consistency should be used to more accurately identify diarrhea in calves.

Key words: hide cleanliness, diarrhea, veal calf, fecal consistency

\section{Short Communication}

Diarrhea is a common disease affecting calves (USDA, 2007; Bähler et al., 2012), causing significant morbidity and mortality (Svensson et al., 2006; Pardon et al., 2013; Windeyer et al., 2014). It can also greatly limit the genetic potential of the calf by negatively affecting ADG (Donovan et al., 1998; Windeyer et al., 2014), age at first calving (Heinrichs et al., 2005), first-lactation milk production (Heinrichs et al., 2005), as well as carcass traits in milk-fed veal calves (Pardon et al., 2013).

To reduce the short- and long-term consequences associated with diarrhea, immediate identification and rehydration of affected calves is crucial before the calves developing clinical signs of dehydration (McGuirk, 2008). An accurate method used to identify calves with diarrhea is to score feces based on consistency. This is accomplished by stimulating calves to defecate and scoring the fecal sample on a 4-point scale (McGuirk, 2008; Curtis et al., 2016; Gomez et al., 2017). Despite this method being a gold standard for identifying diarrhea, it can be time-consuming, inconvenient, and stressful to the calf. The feces can also be scored on bedding substrate; however, this is not feasible in most veal facilities, as the calves are housed on slatted flooring. As an alternative, some calf health studies have used a hide cleanliness score as an indicator of diarrhea (Jorgensen et al., 2017). Hughes (2001) proposed that the leading cause of abnormal hide cleanliness is diarrhea, although conceded that cleanliness may be influenced by many factors such as housing or bedding style, stocking density, and ventilation. Advantages to using hide cleanliness to identify diarrhea include reduced time and ease of diagnosis, improved hygienic measures, and less stress to the calf. 
The objective of our study was to validate the use of hide cleanliness against a gold standard of fecal consistency to identify diarrhea in calves upon arrival at a veal calf facility. In addition, the number of days with an abnormal cleanliness score was compared with total number of days with an abnormal fecal consistency to determine the correlation of multiple abnormal scores over a 3-d period.

This study was conducted in cooperation with a milkfed veal producer and in accordance with the University of Guelph Animal Care Committee requirements (Animal Use Protocol: \#3453). A total of 452 calves were evaluated for hide cleanliness and fecal consistency immediately upon arrival at a milk-fed veal calf facility, and 222 of those calves were monitored for a total of 3 consecutive days after arrival. The calves scored arrived from different sources and were not all housed in the same location. A total of 12 rooms were used to house the scored calves. The age of these calves at arrival was unknown but would likely be a week of age or less. Due to logistic reasons, all calves could not be followed for all $3 \mathrm{~d}$. Calves were housed individually on slatted stalls and were rectally stimulated to encourage defecation; fecal samples were scored for fecal consistency. Fecal consistency was scored on a scale of 0 to 3 , where fecal score $0=$ normal consistency, $1=$ semi-formed or pasty, $2=$ loose feces, and $3=$ watery feces; calves with a fecal score of 2 or 3 were classified as positive for diarrhea (McGuirk, 2008). Hide cleanliness [adapted from Panivivat et al. (2004) and Sutherland et al. (2014)] was also scored on a scale of 0 to 3 , where $0=$ clean thighs and body with little to no manure on lower legs; $1=$ tail head region and back end of calf are soiled with manure; 2 = tail head region, back end of calf, and thighs or legs are soiled with manure; and $3=$ tail head region, back end of calf, thighs, and legs are soiled with manure. All calves were scored by 1 of 2 observers, both trained for consistency by a veterinarian. If an observer was evaluating a calf, both the fecal consistency and hide cleanliness score were completed by that observer. However, different observers may have evaluated the calves when observed for more than $1 \mathrm{~d}$.

For the purposes of sample size calculations a priori, the prevalence of diarrhea (fecal consistency $\geq 2$ ) at arrival was expected to be $15 \%$ (Renaud et al., 2018), and the sensitivity and specificity of the cleanliness scoring as a test in identifying calves with diarrhea were predicted to be 80 and $85 \%$, respectively. Utilizing the method described by Buderer (1996) and assuming the clinically acceptable width of the $95 \%$ confidence intervals for sensitivity and specificity was to be no larger than $10 \%$, the sample size required was determined to be 410 calves.
All statistical analyses were completed using Stata 13 (StataCorp LP, College Station, TX). Data were imported from Microsoft Excel (Microsoft Corp., Redmond, WA) into Stata 13 and checked for completeness. A weighted kappa was used to determine the agreement between the 2 scales on the day of arrival, accounting for the agreement that could occur due to chance (Dohoo et al., 2009). The kappa used ratings weighted by 1.00, $0.67,0.33$, and 0 and allowed partial agreement to be considered, which is important for the continuous scales used in our study. A nonparametric receiver operating characteristic (ROC) curve was generated to compare cleanliness and fecal scores and to determine the sensitivity and specificity of hide cleanliness to classify cases of diarrhea identified using fecal scoring (Dohoo et al., 2009). Fecal score was the classification variable, and was dichotomized to represent diarrhea (score of $\geq 2$ ) and nondiarrhea (score of $<2$; McGuirk, 2008). The cut-point for cleanliness score was selected to optimize sensitivity and specificity to limit the effect of both false-positive and -negative diagnoses (Florkowski, 2008). To evaluate the correlation over multiple days with abnormal scores, simple linear regression analysis was conducted with total days with abnormal cleanliness score ( $\geq 1$ cleanliness score) as the predictor and total days with abnormal fecal score ( $\geq 2$ fecal score) as the outcome of interest. The assumptions of normality and homoscedasticity of the residuals were tested using a Shapiro-Wilks test and Cook-Weisberg test.

The weighted kappa (k) calculated on 452 calves at arrival resulted in a $\mathrm{k}$ value of 0.22 , indicating fair agreement; a $\mathrm{k}$ value of 0 would indicate no agreement above what is expected by chance and a $\mathrm{k}$ value of 1 would indicate perfect agreement (Landis and Koch, 1977; Dohoo et al., 2009). The ROC curve for these data had an area of 0.66 under the curve, which indicates the probability that a randomly selected positive individual has a greater score than a randomly selected negative individual (Dohoo et al., 2009). Assuming the optimal cut-point is the point where sensitivity and specificity are at a maximum, the cut-point for cleanliness score was determined to be a score of $\geq 1$, which correctly classified $64 \%$ of calves (Dohoo et al., 2009). This cut-point generated a sensitivity of $67 \%$, which is the true positive rate, and indicates the proportion of calves with diarrhea that were given an abnormal cleanliness score on arrival. The cut-point of $\geq 1$ had a specificity of $63 \%$, which is the true negative rate, and indicates the proportion of calves that did not have diarrhea and were given a normal cleanliness score at arrival (Florkowski, 2008).

Using this cut-point of $\geq 1$ on the cleanliness scale, 188 of 452 calves (42\%) at arrival would be diagnosed 
Table 1. Numbers of calves with positive and negative cleanliness and fecal scores on arrival at a milk-fed veal facility

\begin{tabular}{|c|c|c|c|}
\hline Item & $\mathrm{CS}+{ }^{1}$ & $\mathrm{CS}_{-}{ }^{2}$ & Total \\
\hline $\begin{array}{l}\mathrm{FS}^{3}{ }^{3} \\
\mathrm{FS}^{4}{ }^{-1} \\
\text { Total }\end{array}$ & $\begin{array}{r}52 \\
136 \\
188\end{array}$ & $\begin{array}{r}26 \\
238 \\
264\end{array}$ & $\begin{array}{r}78 \\
374 \\
452\end{array}$ \\
\hline
\end{tabular}

${ }^{1}$ Cleanliness score positive: score $\geq 1$.

${ }^{2}$ Cleanliness score negative: score $<1$.

${ }^{3}$ Fecal score positive: score $\geq 2$.

${ }^{4}$ Fecal score negative: score $<2$.

with diarrhea; however, using the gold standard of fecal consistency to identify diarrhea (fecal score $\geq 2$ ), only 78 calves $(17 \%)$ were diagnosed (Table 1 ). False-positives (healthy calves that test positive) were calculated by 1 minus specificity, and false-negatives (diseased calves that test negative) were calculated by 1 minus sensitivity, giving values of 36 and $33 \%$, respectively (Florkowski, 2008; Dohoo et al., 2009). Of the 222 calves that were followed for $3 \mathrm{~d}$ (Figure 1), 154 calves $(69 \%)$ did not have any days with abnormal fecal scores $(\geq 2)$, whereas only 77 calves (35\%) did not have any days with abnormal cleanliness scores $(\geq 1)$. Calves were more likely to have more days with abnormal hide cleanliness, with 91 calves (41\%) having an abnormal cleanliness score for at least $2 \mathrm{~d}$, compared with only 21 calves $(9 \%)$ having an abnormal fecal score for at least 2 d. Figure 1 shows that once calves achieve an abnormal cleanliness score they are more likely to maintain this level.

A linear regression of total days with abnormal fecal score against total days with abnormal cleanliness score gave a coefficient of determination value of 0.1 . This indicates poor correlation between the 2 scales and that the model containing total days with an abnormal

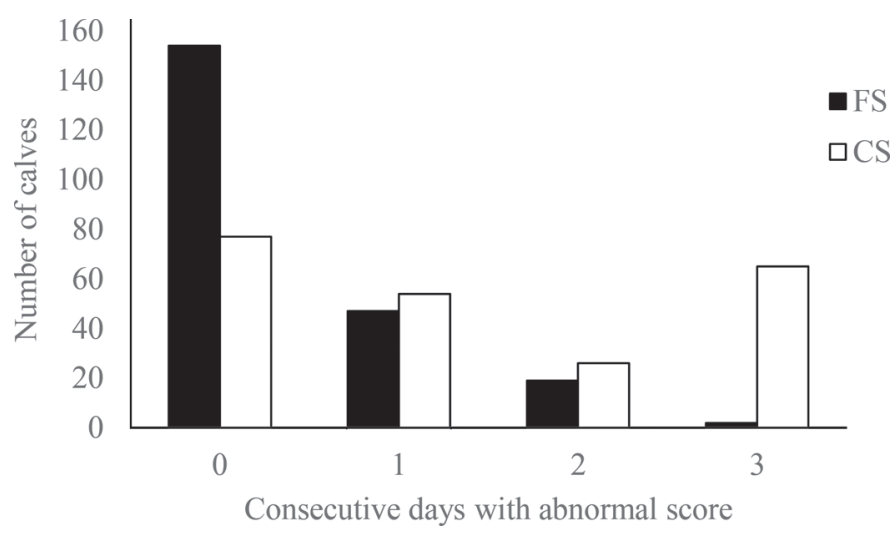

Figure 1. Consecutive days with abnormal fecal and cleanliness scores for calves with $3 \mathrm{~d}$ of data. $\mathrm{FS}=$ fecal score (abnormal if $\geq 2$ ); $\mathrm{CS}=$ cleanliness score (abnormal if $\geq 1$ ). cleanliness score only explains $10 \%$ of the variation in total days with abnormal fecal score. Thus, the number of days with abnormal cleanliness score is not associated with the number of days with diarrhea (Dohoo et al., 2009). The lack of correlation between the scales and the increased likelihood for calves to have consecutive days with abnormal cleanliness scores supports the hypothesis that cleanliness maybe more related to bedding material housed on before arrival than fecal score (Panivivat et al., 2004). Monitoring of the calves for additional days after arrival would be required to draw more conclusions.

Ours is the first study to evaluate the utility of cleanliness scoring as a method to identify calves with diarrhea at the time of arrival at a milk-fed veal calf facility. It demonstrates that cleanliness scoring is not a useful system to identify calves with diarrhea and calves with longer bouts of diarrhea do not have worsening cleanliness scores. A potential limitation of our study was the inability to calculate interobserver agreement between the 2 observers. Due to the subjective nature of the scoring methods, this may have decreased the level of agreement between the 2 scoring methods.

With diarrhea being responsible for a high proportion of mortality in veal calves (Bähler et al., 2012) and dairy heifers (Windeyer et al., 2014), it is important to use methods to identify calves with diarrhea early and provide timely, appropriate interventions (McGuirk, 2008). However, as most calves enter veal and dairy heifer rearing facilities with little or no historical information, it is difficult to identify calves with diarrhea upon entry. Calf cleanliness score can be completed quickly at arrival and had fair agreement with fecal scoring, but the sensitivity and specificity of cleanliness scoring were poor when compared with a fecal consistency of $\geq 2$. With the large number of false-negatives generated when using the cleanliness scoring, many calves with diarrhea would be missed and may not be provided with an appropriate intervention before becoming clinically dehydrated. Based on these results, cleanliness scoring cannot be recommended for assessment of diarrhea.

Cleanliness scoring is used by some welfare programs and is reported as the method of choice as a marker for diarrhea in some published studies (Jorgensen et al., 2017). Although a relatively easy procedure to conduct, it does not reliably identify calves with diarrhea, as determined by the current study, nor is it useful for identifying diarrhea at the herd level (Klein-Jöbstl et al., 2014). Thus, caution needs to be used in the interpretation of the presence of abnormal cleanliness. The cleanliness score is likely more of a reflection of the type of bedding used to house calves at the dairy farm (Panivivat et al., 2004) and could potentially describe 
the comfort level that the calf is experiencing before arrival at the growing facility.

We showed that it is not appropriate to identify diarrhea in calves on arrival at a veal calf facility based on hide cleanliness. Neither single nor consecutive observations of hide cleanliness are accurate representations of cases of diarrhea in calves. To ensure cases of diarrhea are not incorrectly diagnosed or missed, fecal consistency scoring should be used to identify diarrhea in calves. Although these results are likely applicable to most dairy farms, factors, such as cleanliness and stocking density of the transport vehicle, may affect the ability to extrapolate these results to the dairy industry.

\section{ACKNOWLEDGMENTS}

We thank Veal Farmers of Ontario (Guelph, ON, Canada), Dairy Farmers of Ontario (Mississauga, ON, Canada), Grober Nutrition (Cambridge, ON, Canada), and the Ontario Ministry of Agriculture and Rural Affairs for funding this project. We also thank the milkfed veal calf facility for allowing us to conduct this trial.

\section{REFERENCES}

Bähler, C., A. Steiner, A. Luginbühl, A. Ewy, H. Posthaus, D. Strabel, T. Kaufmann, and G. Regula. 2012. Risk factors for death and unwanted early slaughter in Swiss veal calves kept at a specific animal welfare standard. Res. Vet. Sci. 92:162-168. https://doi .org/10.1016/j.rvsc.2010.10.009.

Buderer, N. M. F. 1996. Statistical methodology: I. Incorporating the prevalence of disease into the sample size calculation for sensitivity and specificity. Acad. Emerg. Med. 3:895-900. https://doi.org/10 $.1111 / \mathrm{j} .1553-2712.1996 . t b 03538 . x$.

Curtis, G. C., C. Argo, D. Jones, and D. Grove-White. 2016. Impact of feeding and housing systems on disease incidence in dairy calves. Vet. Rec. 179:512-517. https://doi.org/10.1136/vr.103895.

Dohoo, I., W. Martin, and H. Stryhn. 2009. Veterinary Epidemiologic Research. 2nd ed. Screening and Diagnostic Tests. VER Inc., Charlottetown, Prince Edward Island, Canada.

Donovan, G. A., I. R. Dohoo, D. M. Montgomery, and F. L. Bennett. 1998. Calf and disease factors affecting growth in female Holstein calves in Florida, USA. Prev. Vet. Med. 33:1-10. https://doi.org/ 10.1016/S0167-5877(97)00059-7.

Florkowski, C. M. 2008. Sensitivity, specificity, receiver-operating characteristic (ROC) curves and likelihood ratios: Communicating the performance of diagnostic tests. Clin. Biochem. Rev. 29:S83-S87.
Gomez, D. E., L. Arroyo, M. Costa, L. Viel, and J. Weese. 2017. Characterization of the fecal bacterial microbiota of healthy and diarrheic dairy calves. J. Vet. Intern. Med. 31:928-939. https://doi .org/10.1111/jvim.14695.

Heinrichs, A. J., B. Heinrichs, O. Harel, G. Rogers, and N. Place. 2005. A prospective study of calf factors affecting age, body size, and body condition score at first calving of Holstein dairy heifers. J. Dairy Sci. 88:2828-2835.

Hughes, J. 2001. A system for assessing cow cleanliness. In Pract 23:517-524.

Jorgensen, M., A. Adams-Progar, A. de Passille, J. Rushen, S. Godden, H. Chester-Jones, and M. Endres. 2017. Factors associated with dairy calf health in automated feeding systems in the upper Midwest United States. J. Dairy Sci. 100:5675-5686. https://doi .org/10.3168/jds.2016-12501.

Klein-Jöbstl, D., M. Iwerson, and M. Drillich. 2014. Farm characteristics and calf management practices on dairy farms with and without diarrhea: A case-control study to investigate risk factors for calf diarrhea. J. Dairy Sci. 97:5110-5119. https://doi.org/10.3168/ jds.2013-7695.

Landis, J. R., and G. G. Koch. 1977. The measurement of observer agreement for categorical data. Biometrics 33:159-174. https://doi .org $/ 10.2307 / 2529310$

McGuirk, S. M. 2008. Disease management of dairy calves and heifers. Vet. Clin. North Am. Food Anim. Pract. 24:139-153. https://doi .org/10.1016/j.cvfa.2007.10.003.

Panivivat, R., E. Kegley, J. Pennington, D. Kellogg, and S. Krumpelman. 2004. Growth performance and health of dairy calves bedded with different types of materials. J. Dairy Sci. 87:3736-3745. https://doi.org/10.3168/jds.s0022-0302(04)73512-2.

Pardon, B., M. Hostens, L. Duchateau, J. Dewulf, K. De Bleecker, and P. Deprez. 2013. Impact of respiratory disease, diarrhea, otitis and arthritis on mortality and carcass traits in white veal calves. BMC Vet. Res. 9:79. https://doi.org/10.1186/1746-6148-9-79.

Renaud, D. L., T. Duffield, S. Leblanc, D. Haley, and D. Kelton. 2018. Risk factors associated with mortality at a milk-fed veal calf facility: A prospective cohort study. J. Dairy Sci. 101:2659-2669.

Sutherland, M. A., G. M. Worth, and M. Stewart. 2014. The effect of rearing substrate and space allowance on the behaviour and physiology of dairy calves. J. Dairy Sci. 97:4455-4463. https://doi.org/ 10.3168/jds.2013-7822.

Svensson, C., A. Linder, and S. Olsson. 2006. Mortality in Swedish dairy calves and replacement heifers. J. Dairy Sci. 89:4769-4777. https://doi.org/10.3168/jds.S0022-0302(06)72526-7.

USDA. 2007. Dairy 2007, Part I: Reference of Dairy Cattle Health and Management Practices in the United States, 2007. USDA-APHISVS, CEAH, \#N480.1007. National Animal Health Monitoring System, Fort Collins, CO.

Windeyer, M. C., K. Leslie, S. Godden, D. Hodgins, K. Lissemore, and S. LeBlanc. 2014. Factors associated with morbidity, mortality, and growth of dairy heifer calves up to 3 months of age. Prev. Vet. Med. 113:231-240. https://doi.org/10.1016/j.prevetmed.2013 .10.019. 